\title{
Physical Modeling on Time Domain Induced Polarization (TDIP) Response of Metal Mineral Content
}

\author{
Yatini $^{1^{*}}$, Djoko Santoso ${ }^{2}$, Agus Laesanpura ${ }^{2}$, Budi Sulistijo ${ }^{3}$ \\ ${ }^{1}$ Geophysics Engineering, Faculty of Mineral Technology, Universitas Pembangunan Nasional "Veteran" \\ Yogyakarta SWK No.104 North Ring Road Street Condong Catur 55283, Yogyakarta \\ ${ }^{2}$ Department Geophysics Engineering, FTTM- ITB, Ganesha Street 10, Bandung. \\ ${ }^{3}$ Mining Department, FTTM- ITB. Ganesha Street 10, Bandung. \\ *Email: jeng_tini@upnyk.ac.id
}

\section{ABSTRACT}

The Induced Polarization (IP) methods is an extension of resistivity method by adding ability of the ground in storing electrical charge. One of the measurement technique is done in time domain, hereinafter referred to as Time Domain Induced Polarization (TDIP). TDIP responses measured on the surface are affected by the physical properties of the subsurface. Research in TDIP response modeling studies is performed to obtain a quantitative relationship between response to metallic mineral content at subsurface. The relationship can be obtained by forward and physical modelling. The forward modeling produces a curve that connects TDIP response to the subsurface parameters and an array. The laboratory-scale physical model is performed on the sand-box size $(200 \times 100 \times 70) \mathrm{cm}^{3}$ by varying iron-ore content in a sphere target. TDIP response measurements on physical models is done using Dipole-dipole and Wenner configuration. The relationship between the TDIP response and metal mineral content is obtained by comparing the results of measurements on physical modeling and forward modelling. There is good appropriatement between the theoretical curves and measuring results of the physical modelling. The greater of iron-ore content on the target, increasing in the TDIP response.

Keywords: TDIP response, chargeability, resistivity, forward and physical modelling

\section{INTRODUCTION}

IP method is one of Geophysics method that widely used for the exploration of base metal since last 60 years. This method is an extension of the resistivity method by adding parameters ability to store electrical charge. The Measurement of the IP method can be done in the domain of time, frequency and phase. Measurement in time domain known as Time Domain Induced Polarization (TDIP). Ability to store charge in TDIP expressed by chargeability parameters. Chargeability can be used to indicate the presence of metallic minerals in the subsurface.

TDIP studies on physical models were originally performed ${ }^{[1]}$ which did soundings on polarisable alluvial bedding beneath a layer of sand. IP response dropped to a minimum value, when spacing of Wenner electrodes is longer than the thickness of the polarisable layer. The measurements of TDIP response on finite body model of graphite on sand medium was used Gradien configuration varied in slope and depth ${ }^{[2]}$. The making of physical models to determine the depth of penetration of varies electrode was done ${ }^{[3]}$. The sand-box is contained of two and three layers of sand and soil, and measuring the resistivity. 
The resistivity measurement of graphite blocks placed in the water medium on the sand-box sized $(100 \times 200 \times 100) \mathrm{cm}^{3}$, modeled dike ${ }^{[4]}$. The resolution of resistivity anomalies with varied electrode configuration is obtained by comparing the theoretical and experimental results. The TDIP response measure on different graphite sphere with the water medium ${ }^{[5]}$. Physical models to determine the depth penetration were using sphere and cylindrical graphite placed in the sand-box. The measurement was done by sounding with two frequencies ${ }^{[6]}$. The results are penetration depth dependent on targets shape and current electrode distance, while the frequency is unaffect. Physical model studies can be carried out with four electrode set-up keeping the target physically in the host medium of the model sand-box and measuring the responses at different transition parameters ${ }^{[1,7,8]}$.

The relationship between the metallic mineral content in the subsurface and measured TDIP response at the surface is not known with certainty. In this study, an attempt to obtain the relationship is done by modeling studies. Modeling studies in this research include forward and physical modeling using a laboratory scale at sand-box size $(200 \times 100 \times 70) \mathrm{cm}^{3}$. The results of TDIP response at the insulator sphere target are adequate ${ }^{[9]}$. The foreward modeling resulting theoretical curves of TDIP response. This curve connect TDIP response toward geometry and arrays used. Res2Mod is used to obtain the curve of theoretical models ${ }^{[10]}$ and displayed with Microsoft Excel Graphycs. Physical modeling is intended to obtain measurement results TDIP response to subsurface conditions are known with certainty. Groundwater is used as the host medium and the sphere with iron-ore content varied as the target. Mixture of the target was using iron-ore, quartz sand and cement. The relationship between TDIP response and metalic mineral content was obtained by curve matching analysis of theoretical and measurement results on physical modelling.

\section{METHODS}

\section{Forward Modelling}

The TDIP response caused by the sphere below the surface (Figure 1) is obtained by solving the Laplace equation. The boundary condition is applied to obtain apparent resistivity equation as a function of sphere's radius and depth, resistivity of sphere medium and configuration used. TDIP response is obtained by derivating such as the definition of [11, 12]. Forward modeling is conducted to obtain the theoretical value of the TDIP response whereas subsurface parameters are known. The response value of $n=1$ to 8 are created to curves and pseudosection theoretically.
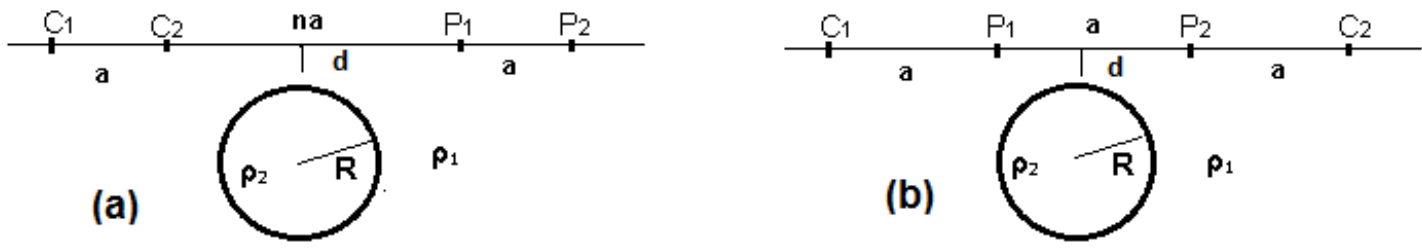

Figure 1. Simple sphere geometry model, with (a) Dipole-dipole and (b).Wenner configuration. 


\section{Physical Modeling Laboratory Scale}

Physical modeling is done on the sand-box size (200x100x70) $\mathrm{cm}^{3}$ and TDIP response measured at surface. The groundwater is used as a host medium which approximate homogeneous isotropic so that measuring results is quite good ${ }^{[5,13]}$. Target is sphere with radius $5 \mathrm{~cm}$ with iron-ore content of $20 \%, 40 \%, 70 \%$ and $80 \%$ respectively. Target mixture consists of iron-ore, quartz sand and cement. One of the minerals in iron-ore is magnetite which has high chargeability and low resistivity. The quartzite minerals at quartz-sand is highly resistant, hence always present in almost rocks. This mixture is ideal to explain how the role of metallic mineral deposits on the TDIP response.

Data aquisition of TDIP on sand-box using the stainless stell sticks with diameter of $1 \mathrm{~mm}$ as current electrode and porouspot as potential electrode. The use of these electrodes resulted in a good enough data ${ }^{[14]}$. The porouspot electrodes also used to measure IP response at the sample ${ }^{[15,16]}$. TDIP response measurements on physical modeling was using the main tool IPMeter Syscal IRIS Instruments 568 series that can be used to measure the resistivity and chargeability. The equipment setting to obtain data on physical modeling is shown in figure 2 .

IP data acquisition is very similar to resistivity data acquisition. All IP instruments also record electrical resistivity concurrently. However, much smaller voltages are measured in the IP method, requiring additional consideration during data acquisition. It is important to emphasize that all IP instruments also record the resistivity magnitude in addition to the IP response (chargeability). The resistivity is an important parameter in the interpretation of IP data.

TDIP data aquisition is done by injecting current trough $\mathrm{C}_{1} \mathrm{C}_{2}$ then measuring potential at $\mathrm{P}_{1} \mathrm{P}_{2}$. A current send and afterwards off, subsequently terminated immediately zero potential, but in reality it will go to zero for a few seconds or minutes. Chargeability will be obtained by measuring the decay time. Dipole-dipole and Wenner configuration are used to know the resistivity distribution and chargeability below the surface.

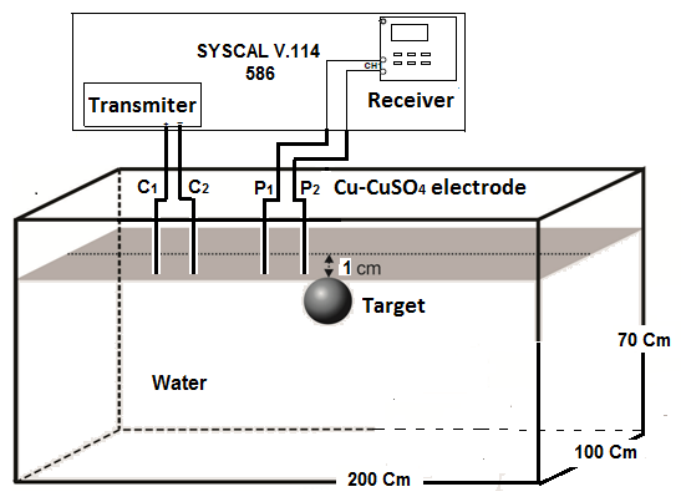

Figure 2. Setting of the instrument used to the physical modeling 


\section{Induced Polarization Parameter}

In simple term, IP response reflects the degree which the subsurface is able to store electrical charge, analogous to a capacitor ${ }^{[17]}$. This polarization occurs at the interface between (1) a metal and a fluid (electrode polarization), and (2) a non-metal (e.g. silica or clay minerals) and a fluid (traditionally called membrane polarization). Polarization is resulted from a redistribution of ions along such interfaces following application of an electric current. Upon current termination, ions relax to the equilibrium condition. This diffusion-controlled relaxation is equivalent to a residual current flow (as observed during discharge of a capacitor) and is the source of the subsurface IP response. The IP method measures the magnitude of this polarization. In contrast, the resistivity method measures the magnitude of conduction provided by both electrolytic and surface conduction (enhanced in the presence of clay minerals). Electrode polarization generally produces a larger IP response than membrane polarization ${ }^{[18]}$.

In doing so, a low frequency current or direct current (DC) is injected at two current electrodes, while the potential difference is measured on the potential electrode. The square wave generated current electrodes and the signal received at the potential electrodes (Figure.3). When the current is disconnected, potential will immediately zero. However, in IP measurement, the potential will be zero for several time interval, this is called potential decay. Potential decay is due to the polarization in the subsurface medium.

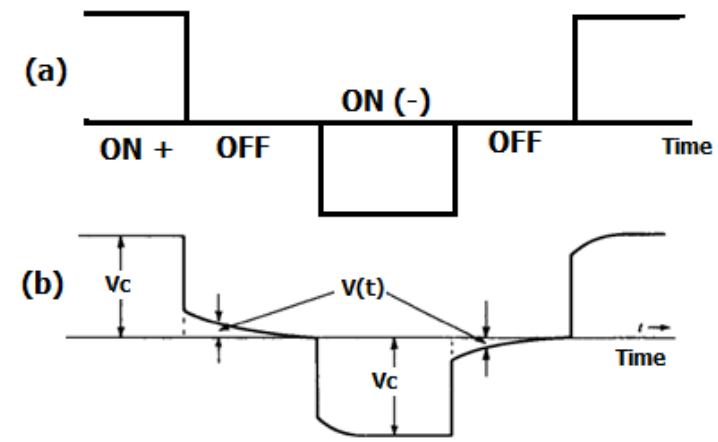

Figure 3. (a) Current waveform : square- wave is generated low frequency. (b) Time domain IP signal received showing measured parameters.

At the time domain, there is most common measurement is the chargeability defined as ${ }^{[19]}$ :

$$
m=\frac{1}{V_{0}} \int_{t_{1}}^{t_{2}} V_{p}(t) d t
$$

Where $V_{p}(t)$ is residual voltage integrates over time window defined between times $t_{1}$ and $t_{2}$ after termination of an applied current. $V_{o}$ is the measured voltage at some time during application of the current. The unit of chargeability are quoted as millivolt per volt (mV/V) and is the most commonly used quantity in time domain IP measurement. When $V_{p}(t)$ and $V_{o}$, have the same units, the chargeability $m$ is in millisecond. 


\section{RESULTS AND DISCUSSION}

The solution of Laplace equation generates TDIP response caused by objects that are below the surface. TDIP response can be expressed as a curve which describes the change in lateral TDIP response. In obtaining the curves of sphere at subsurface models using Res2Dmod ${ }^{[10]}$. Display curve used Microsoft Excel Graphycs. Theoretical curve from forward modeling results at sphere radius $5 \mathrm{~cm}$ and depth of $1 \mathrm{~cm}$ with Dipole-dipole and Wenner in the figure (4).

Forward Modeling with Res2Dmod must enter of resistivity and chargeability for target and host medium. This value is obtained from direct measurements on samples and groundwater used as the host medium (Table 1).

Forward modeling result in curve that describes the relationship TDIP response to subsurface parameters, i.e. the depth, the sphere radius and resistivity of the target and host medium. This curve can be used as consideration for the selection of appropriate parameters applied on a laboratory scale physical modeling so that the optimum result can be obtained.

In this case, the greatest response is at $n=1$ for Dipole-dipole and Wenner. The curve comparison spaced at the same depth was also made. This curve can be used to select the appropriate spaces on the desired depth target. If it applied to the physical modelling, the results will be optimum. In case the sphere is at a depth of $1 \mathrm{~cm}$, the best spacing is $5 \mathrm{~cm}{ }^{[20]}$.
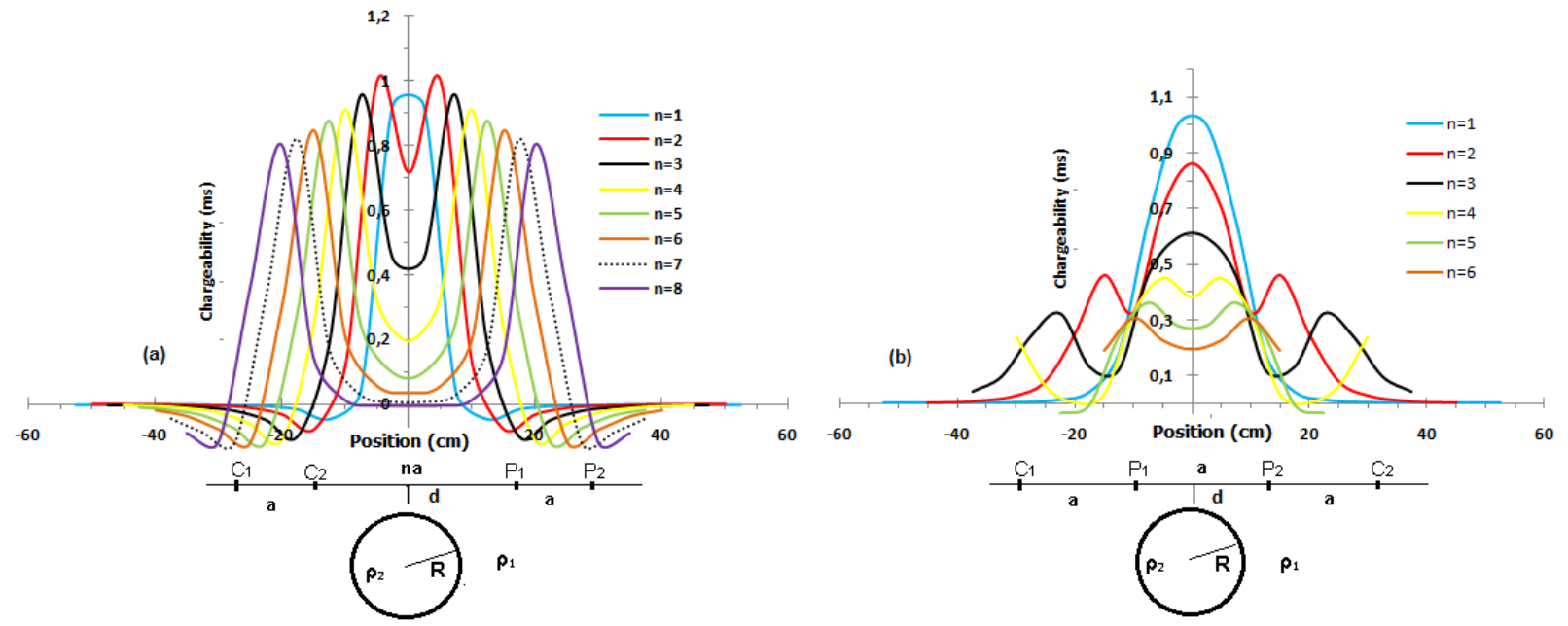

Figure 4. (colour online) Theoretical TDIP response normalized subsurface sphere with radius $\mathrm{R}=5 \mathrm{~cm}$ and a depth $d=1 \mathrm{~cm}$. (a).n=1-8 for Dipole-dipole and (b). $n=1-6$ for Wenner configuration.

\section{Physical Modeling}

In this study an attempt to gain influence on the response of metallic mineral deposits TDIP is performed by measurement laboratory scale physical modelling. Chargeability and resistivity of water as a host medium are measured at the time of measurement (Table I). Chargeability and resistivity measured on physical modeling surface are apparent value. The magnitude depends on the resistivity of target and host medium, dimensions and depth of the target and 
configuration used. Efforts to determine the relationship of metallic mineral deposits on TDIP response is to match the curve of the measuring results against theoretical curves.

In this study, laboratory-scale physical modeling on glass sand-box sized $(200 \times 100 \times 70) \mathrm{cm}^{3}$ is the best when using space $5 \mathrm{~cm}$. Dipole-dipole uses $\mathrm{n}=1-5$ and $\mathrm{n}=1-4$ for Wenner. Resistivity and chargeability of the targets and host medium are shown in Table (I). Curve resulted on chargeability measuring on physical modeling with a the iron-ore content of target $20 \%, 40 \%$, $70 \%$ and $80 \%$ respectively, Wenner configuration is shown in figure (5). The amplitude curve measurement results showed a positive correlation with the levels. Average amplitude changes with increasing level of measurement targets. The greater level of the measuring targets, the greater measuring results chargeability amplitude.

Table 1. Resistivity and TDIP response value on the target and the host medium is used in forward and physical modeling

\begin{tabular}{|c|c|c|c|c|c|}
\hline \multirow[t]{2}{*}{ No } & \multirow{2}{*}{$\begin{array}{c}\text { Iron-ore } \\
\text { content } \\
(\%)\end{array}$} & \multicolumn{2}{|c|}{ Targets } & \multicolumn{2}{|c|}{ Host Medium } \\
\hline & & $\rho($ Ohm-m) & m (ms) & $\rho($ Ohm-m) & m (ms) \\
\hline 1 & 20 & 16.74 & 1.88 & 36.63 & 0 \\
\hline 2 & 40 & 16.68 & 2.43 & 36.63 & 0 \\
\hline 3 & 70 & 47.02 & 3.12 & 36.63 & 0 \\
\hline 4 & 80 & 43.22 & 4.71 & 36.63 & 0 \\
\hline
\end{tabular}
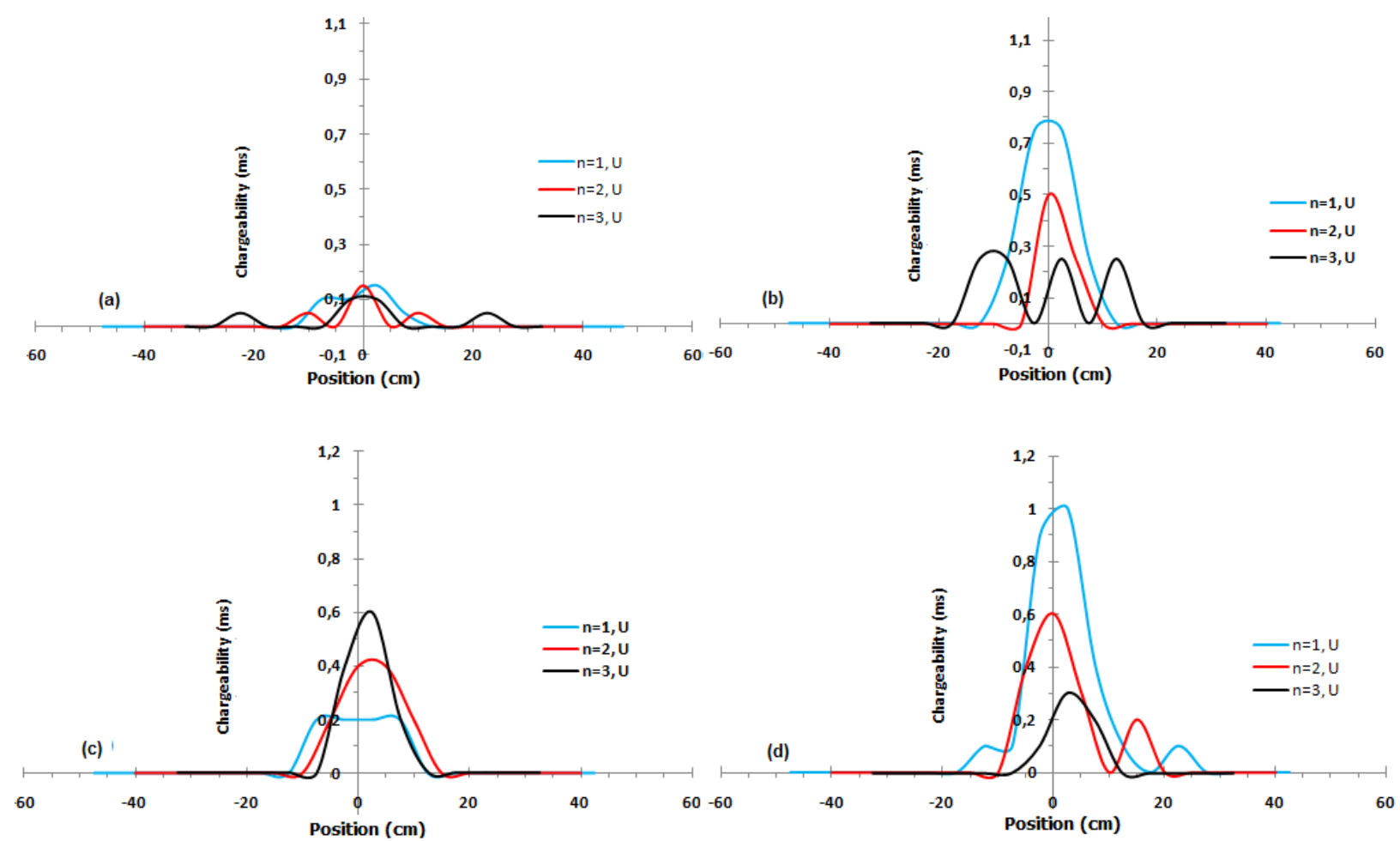

Figure 5. (Colour online) TDIP response measuring curve of sphere in subsurface with radius $\mathrm{R}=5 \mathrm{~cm}$ and a depth of $1 \mathrm{~cm}, 5 \mathrm{~cm}$ spacing Wenner configuration, $\mathrm{n}=1-3$, the iron-ore content of (a) $20 \%$, (b) $40 \%,(c) 70 \%$ and (d) $80 \%$ 
One of standard procedure of data processing in IP method is to perform the inversion. In this case, it carried on theoretical data, and the data measuring TDIP on physical modelling. Inversion used Res2DInv ${ }^{[21]}$. The inversion results show the distribution of true resistivity and true chargeability on true depth. Inversion performed on theoretical data should produce distribution TDIP response parametercorresponding to the geometry of the sphere. In reality that not ever be, because data cutt of $n=1-5$ for Dipole-dipole and $n=1-4$ for Wenner configuration. The resulting depth of the inversion results only up to $6.5 \mathrm{~cm}$ for the dipole-dipole and $9.5 \mathrm{~cm}$ for the Wenner. Inversion results on Dipole-dipole configuration can not cover all parts of the sphere, but for the Wenner entire sphere can be seen. The advantages of physical modeling is the position and geometry of the target is known with certainty, so that it can be seen whether the results quality of the inversion with Res2DInv.

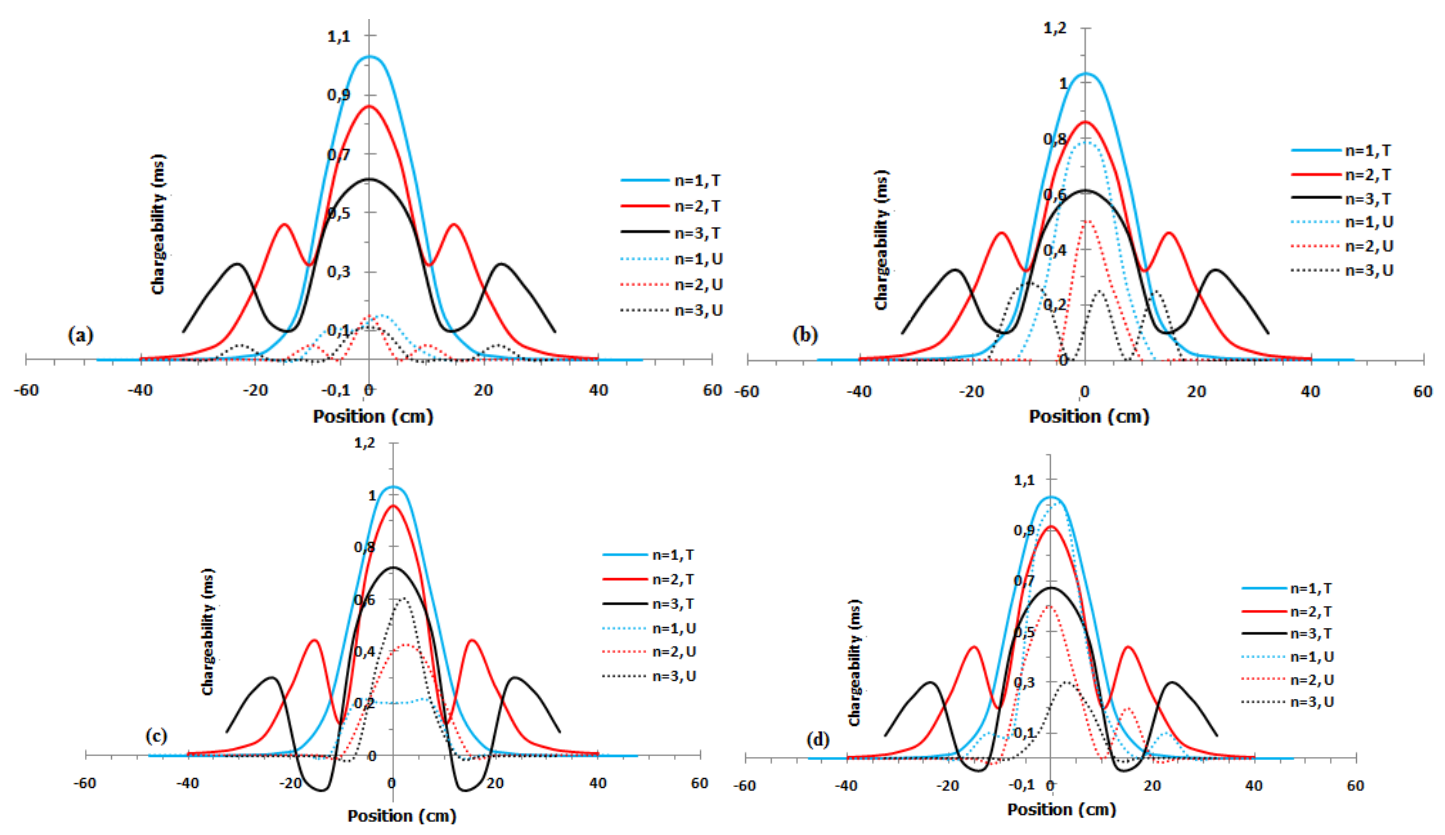

Figure 6. (Colour online) Comparison of theoretical curves TDIP response (continue) and measuring (dashed) at spheres with the iron-ore content of (a)20\%, (b)40\%, (c)70\% and (d)80\% for the Wenner configuration, 5 $\mathrm{cm}$ spacing and $\mathrm{n}=1-3$.

The Matching of theoretical and measurement.

Matching the theoretical and the results measured in this study, is one way to be knew the influence of the magnitude of metallic minerals toward the TDIP response. The resulting curves forward modeling of mathematical calculations in comparison with the curve obtained from the measurement with the same geometry. The purpose of this is to determine the extent of conformity between the theoretical response and the TDIP response measured.

The curve matching of theoretical and measuring is done in space and $\mathrm{n}$ for each configuration used. Comparison to the target sphere iron-ore content of $20 \%, 40 \%, 70 \%$ and $80 \%$, there is a fairly good conformity (figure 6). The greater the levels of iron-ore measuring, the bigger amplitude of the curve. This indicates that there is the influence of iron-ore with TDIP response. Level of iron-ores represent metallic mineral content (Fe-total). The greater of metallic minerals content, increase in the TDIP response. 
The matching of pseudodepth section theoretical and measured performed. Figure (7) is sample with $20 \%$ iron-ore content using Wenner configuration. The difference in average of resistivity and chargeability using Dipole-dipole are $13.58 \%$ and $31.22 \%$, while using Wenner are $11.97 \%$ and $32.29 \%$ respectively.

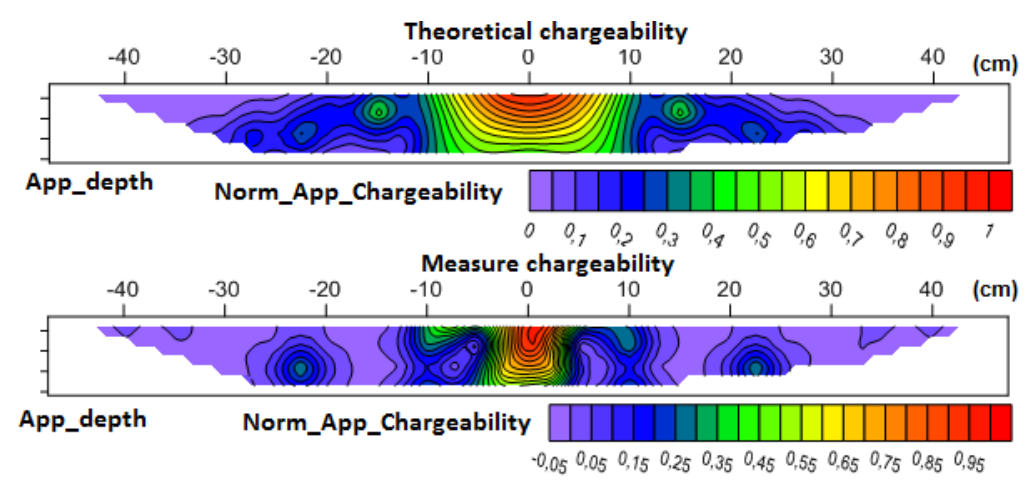

Figure 7. (colour online) Comparison of pseudodepth section theoretically and measuring TDIP response of sphere $20 \%$ iron-ore content, Wenner configuration.

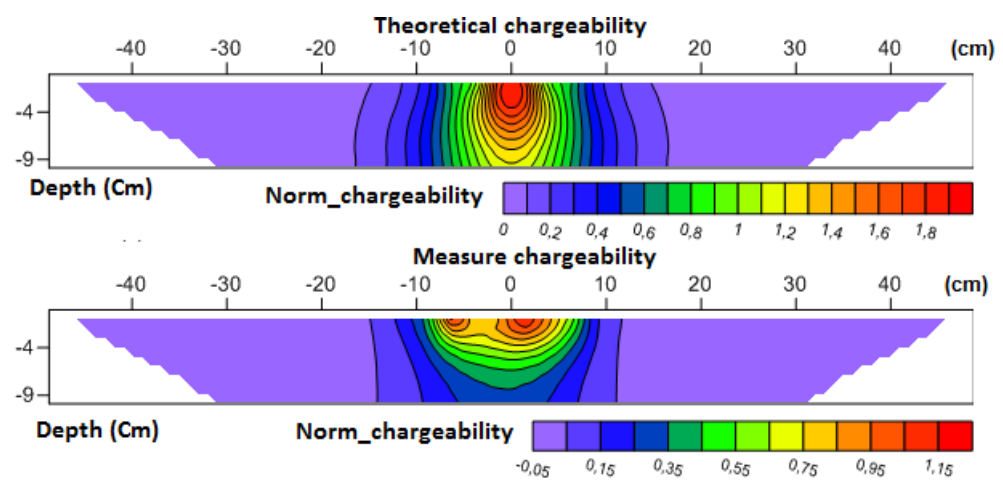

Figure 8. (colour online) Comparison of theoretical inversion results and the results of measured TDIP response on sphere 20\% iron-ore content, Wenner configuration.

Matching on theoretical inversion section and measurement are also performed. Examples of the target of $20 \%$ are shown in figure (8). The difference in average of resistivity and chargeability using Dipole-dipole are $20.81 \%$ and 33.95\%, while using Wenner are $34.0 \%$ and $37.64 \%$ respectively.

In physical modeling there are several things that must be done in order to obtain good results. Equipment, the use of electrodes, the influence of sand-box edge effects, surface polarization effect, the target material and the effect of electromagnetic coupling (EMC), are factors that can cause measurement errors. Data retrieved by injecting current that is sent greater for increasingly electrode distances. The current are reflect by glass walls sand-box and emerge of EMC. The influence of reflection can be removed by applying delay. EMC influence minimized using DC current, applying delay and stacking at the data readout.

The Apparao model's uses a resistivity contrast 0.01, meaning that the host's resistivity 100 times the target or vice versa. In this study the resistivity contrast is 0.45 to 0.85 (Table I). A large resistivity contrast between the host medium and the target is quite difficult to be made. 
The target made of a mixture of iron-ore, quartz sand and cement. The target porosity is $15 \%$ and placed in a sand-box containing of groundwater as host medium. Pore of the targets is what causes the resistivity becomes smaller exponentially ${ }^{[22]}$ so that its value is almost equal to the resistivity of the host medium.

Although Syscal is used as a measurement instrument on a physical models laboratory scale is not idealized, but it is still a good instrument to use because it can measure the resistivity and chargeability from low to high. A current accuracy is $0.01 \mathrm{~mA}$ and potential $0.001 \mathrm{mV}$, but accuracy of chargeability $0.1 \mathrm{~ms}$. This is what causes the error chargeability greater than the resistivity.

\section{CONCLUSION}

Forward modeling result of curve that describes the relationship TDIP response to subsurface parameters. These curves can be used as consideration for the selection of appropriate parameters applied to laboratory scale of physical modelling. There is a good compatibility between the theoretical curve and measuring results. Variations in iron-ore content of $20 \%, 40 \%, 70 \%$ and $80 \%$ indicate that the greater the amplitude curve for Dipole-dipole and Wenner configuration. This indicates the greater the content of metallic minerals in the target, the greater the TDIP response.

\section{ACKNOWLEDGEMENT}

We thanks to Ari and Rizki who helped with the laboratory measurements included in this study and Pak Barko for discuss of measurement desain.

\section{REFERENCES}

1 Vacquier, V., Holmes, C. R., Kintzinger, P. R., \& Lavergne, K. 1957. Prospecting For Groundwater by Induced Electrical Polarization. Geophysics, 22, 660-687.

2 Majumdar, R.K., \& Dutta, S. 1984. Induced Polarization (IP) Time Domain Equipment and Some Model Studies Over Thin Dikes of Finite Strike Extent. Geophysics, 49 (4),291-296.

3 Apparao. A. 1997. Development in Geoelectrical Methods. A.A. Balkema Publs, Old Post Road, Brookfield VT 05036. USA.

4 Apparao, A., Sivarama Sastry, R., \& Sarma S.V. 1996. Depth Detection of Burried Resistive Target with Some Electrode Array in Resistivity Prospecting, Geophysical Prospecting, 43.

5 Sarma, V.S. 2009. Boundary estimation Between Dissemination and Massivity in Mineral Using Physical Model Studies in Induced Polarization (IP). International Workshop on Induced Polarization in Near-Surface Geophysics. Bonn. Germany.

6 Li, J., Liu, J.X., Tong, X.Z., \& Guo, Z.W. 2010. The Physical Modeling Experiments Analysis of the Exploration Depth of Conventional Electric Survey. PIERS Proceedings. Xi'an. China.

7 Apparao, A., \& Roy. A. 1971. Resistivity model experiments II. Geoexploration, 9 (4), 195206.

8 Saydam, A.S., \& Duckworth, K. 1978. Comparison of Some Electrode Arrays for Their IP and Apparent Resistivity Responses over a Sheet-Like Target. Geoexploration, 16, 267-291.

9 Yatini, Santoso, D., Laesanpura, A., \& Sulistijo, B. 2014. Penerapan Moving Average pada Data Polarisasi Terinduksi dalam Domain Waktu (TDIP) Hasil Pemodelan Fisis. Proseding Seminar Nasional Kebumian. Yogyakarta 
10 Loke, M.H. 2002. Rapid 2D Resistivity Forward Modeling Using the Finite-difference and Finite-element methods. Res2Dmod Ver. 3.01 User Manual.

11 Siegel, H.O. 1959. Mathematical Formulation and Type Curve for Induced Polarization. Geophysics, 24, 547-565.

12 Wait, J.R. 1984. Relaxation Phenomena and Induced Polarization. Elsevier Science Publ. Co. Inc. Geoexploration, 22, 107-127.

13 Yatini, Santoso, D., \& Laesanpura, A. 2013. Respon Polarisasi Terinduksi Dalam Kawasan Waktu (TDIP) Pada Medium Air Tanah. Proseding Seminar Nasional Kebumian. Yogyakarta.

14 Yatini, Laesanpura, A. 2013. Influence of Potential's Electrode Selection on Physical Modeling of Time Domain Induced Polarization (TDIP). Case Studies of Homogeneous Isotrop Medium. AIP Conf. Proc. 1554. 1 (2013)

15 Vanhala, H., and Soininen, H. 1995. Laboratory Technigue for Measurement of Spectral IP Response of Soil Samples. Geophysical Prospecting, 43, 454-472.

16 Kiberu, J. 2002. Induced polarization and Resistivity measurements on a suite of near surface soil samples and their empirical relationship to selected measured engineering parameters. Master Thesis. International Institute For Geo-Information Science And Earth Observation Enschede. The Netherlands

17 Sumner, J.S. 1976. Principles of Induced Polarization for Geophysical Exploration. Elsevier Scientific Publ. Co.

18 Slater, L., Lesmes, D. \& Sandberg, S. K. 2000. IP interpretation in environmental investigations: Proceedings of the Symposium on the Application of Geophysics to Engineering and Environmental Problems, 935-944.

19 Ward, S.H. 1990. Resistivity and Induced Polarization Methods. SEG investigation on Geophysics 5 Geotecnical and Enviromental Geophysics, 1, 147.

20 Yatini, Santoso, D., Laesanpura, A., \& Sulistijo, B. 2014. Forward Modeling of Time Domain Induced Polarization (TDIP) Response for Simple Earth Geometries. Proceedings The 5th Annual Basic Science International Conference, 5.

21 Loke. 2003. Electrical Imaging Surveys for Enviromental and Engineering Studies. A practical guide to 2-D and 3-D surveys.

22 Yatini, Santoso, D., Laesanpura, A., \& Sulistijo, B. 2014. Influence of Physical Parameters to Time Domain Induced Polarization (TDIP) Response. AIP Conference Proceedings 1719, 030010 (2016) 